\title{
Géneros para la persuasión en prensa: los editoriales del Diario El País
}

\author{
Dra. Pastora Moreno Espinosa \\ Profesora Titular de Redacción Periodística \\ Universidad de Sevilla
}

\section{RESUMEN}

La prensa en la década de los noventa se ha convertido en periodismo de discusión y debate, en definitiva, en opinión del acontecer político de los últimos años. Los periódicos actuales han devuelto la vida y la vigencia a un género periodístico, el editorial, de gran tradición, uno de los géneros en los que el periodismo español ha conocido sus mejores páginas.

Es el género periodístico que manifiesta el punto de vista del medio sobre un determinado tema de especial relevancia para la actualidad a través de su interpretación y valoración. Es la opinión más autorizada que da el periódico sobre la interpretación de la noticia.

Hoy en día el editorial es la carta de presentación y el elemento de identificación ideológica de cualquier medio de comunicación, especialmente de los escritos, como el diario El País. Aquí radica el interés de esta investigación.

\section{ABSTRACT}

The press in the decade of the ninety has become journalism of discussion and debate, in final, in opinion of the happen politician of the last years. The present newspapers have returned the life and the force to a journalistic kind, the editorial one, of great tradition, one of the kinds in which the Spanish journalism has known its better pages. It is the journalistic kind that manifests the point of view of the mass media upon a determined fear of special importance for the present time through its interpretation and appraisal. It is the most authorized opinion that gives the newspaper upon the interpretation of the news. Nowadays the editorial one is the letter of presentation and the ideological element of identification of any mass media, especially of the writings, as the newspaper The Country. Here it situates the interest of this investigation.

Palabras claves: Periodismo/Debate/Discusión/Identificación/Ideología/Pensamiento/Vigencia.

Key words: Journalism/Debate/Discuss/Identification/Ideology/Mind/Prevailing.

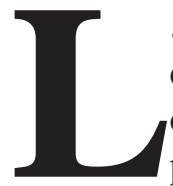

a división entre información y opinión rara vez aparece de forma pura en los textos periodísticos. La mayoría de los géneros periodísticos son complementarios unos de otros. Como la noticia, género informativo por excelencia, complementa al reportaje, del mismo modo los géneros de opinión complementan la información ofrecida por los géneros informativos. 
En la presente investigación analizamos cada una de las modalidades discursivas que definen el editorial como género periodístico de opinión. Prestamos en cada una de ellas especial atención al lenguaje como principal elemento que marca las diferencias entre los distintos géneros.

Los géneros de opinión parten casi siempre de acontecimientos de la actualidad y ofrecen también elementos interpretativos, aunque van más allá y se ocupan de la reflexión profunda que la información de actualidad no puede ofrecer a sus lectores.

Los textos periodísticos de opinión trabajan sobre ideas y deducen consecuencias de unos acontecimientos más o menos actuales. La mayoría de estos discursos van firmados.

Todo texto firmado por un periodista de plantilla o colaborador, en mayor o menor grado, supone un punto de vista que compromete también al periódico y que éste incluye precisamente porque lo considera valioso dentro del marco de objetivos ideológicos que definen su línea editorial.

Por otra parte, los textos de opinión se pueden traducir como actos de representación mental que intentan explicar la realidad de una manera lógicoracional. La persuasión en los textos argumentativos está fundamentada, como en la antigua prensa de opinión, en el prestigio de quien escribe. Se trata de la prueba del argumento de autoridad, garantizada por la sabiduría del experto y rubricada por la influencia del periódico.

Históricamente, a principios del siglo XX había quedado atrás la confianza ciega en las opiniones de la prensa de partido en beneficio del articulista más o menos independiente y ajeno a los intereses inmediatos del juego político. La información y una cierta independencia eran valores en alza, la concepción empresarial del periodismo era un hecho irreversible. En este marco nacen en 1903, $A B C$ y en 1910, El Debate. En Cataluña, el punto de referencia es La Vanguardia, que concebido como órgano del partido liberal en 1881, en manos de Ramón Godó tomaría orientaciones informativas e independientes con tendencia conservadora y monárquica.

Tras la Primera Guerra Mundial, la polémica, el articulismo cruzado y los editoriales intencionados reaparecen con renacida intensidad. El género periodístico de opinión se renueva en la década de los veinte con el articulismo humorístico junto a la historieta y el chiste gráfico. Asimismo las revistas literarias conocen un extraordinario momento. Tal es el caso, por ejemplo, de la Revista de Occidente y La Gaceta Literaria donde escribe parte de los autores que integran la Generación del 27.

En los años treinta, un articulismo comprometido pide la regeneración de España. En esta época, se reproduce con diferentes criterios periodísticos, el ardor opinativo de la prensa del XIX y no sólo en los editoriales. En los numerosos periódicos de estos años colaboran asiduamente las figuras prestigiosas y solventes 
de la vida nacional en todos los aspectos. Los artículos de fondo y los breves ensayos que se publican a diario, alcanzan verdadera categoría literaria. Los acontecimientos sociales y debates parlamentarios suelen ir precedidos de encendidas polémicas en las columnas periodísticas que el público lee y comenta apasionadamente como nunca se ha producido antes ni después. A partir de 1930, se publica más prensa que nunca y con mayores tiradas. El Sol es un buen ejemplo de ello. Esta publicación se convierte en el diario de los intelectuales de primer orden y decenas de millares de lectores.

Llega la República y con ella una ley de prensa que mantiene las tradicionales multas y suspensiones. Una vez más en la historia del periodismo español se crea una Liga defensora de la libertad de prensa, que únicamente no suscriben El Socialista, El Liberal y Luz. A lo largo de estos años las fuertes diferencias ideológicas se fueron acentuando, y esa situación provocó la necesidad de tomar posiciones. De nuevo se alienta el desarrollo del articulismo y por supuesto la necesidad de encontrar medios para poder definir esas posiciones. Como en el siglo XIX, en la primera mitad del XX, comienza a practicarse una prensa con orientación doctrinal y polemista, pobre en información, medios y páginas.

Durante la Guerra Civil hubo fluctuaciones de la prensa debido a que cada zona mostraba la orientación propia de quienes la controlaban. No obstante, las publicaciones del frente populista fueron mayores en número. En el frente de batalla, la prensa combina informaciones oficiales con un articulismo lleno de consignas. El bando populista cuenta con publicaciones como Avance y La Voz del Combatiente. En el bando franquista destacan El Soldado Español o El Alcázar. En definitiva, en uno y otro bando, el articulismo doctrinal está a la orden del día.

En la retaguardia, hay un periodismo de opinión de exigente profundidad y tono más sereno que en La Hora de España. Cultivan autores como José Bergamín, Alberti, Dámaso Alonso, María Zambrano o Machado. El final de la guerra provoca el final de una época de esplendor cultural iniciado en España desde finales del XIX.

Tras la Guerra y sobre todo en el período que media entre la Ley de Prensa de 1938 de Serrano Súñer y la Ley de Prensa e Imprenta de Manuel Fraga en 1966, el panorama en el periodismo de opinión fue básicamente unidireccional. Sólo hay lugar para el discurso oficial y propagandístico que se concentra sobre todo en la prensa del Movimiento, que en 1944 contaba con 37 diarios y 15 revistas.

Hacia 1957, se aprecia un leve cambio en la prensa y paulatinamente queda atrás esa férrea concepción del carácter propagandístico y educativo de la prensa. Los periódicos del Movimiento van incorporando en sus plantillas brillantes colaboradores en temas diversos, en principio, económicos, sociales, deportivos, diluyendo poco a poco en apariencia la dualidad social. En estos años, aparecen en escena articulistas como Eugenio D’Ors, Ramón Gómez de la Serna, Jaime 
Campmany, Cándido, Luis María Ansón, César González Ruano, Guillermo Díaz Plaja o Manuel Santaella.

En década de los sesenta y sobre todo a partir de 1966, la prensa española es el escenario de la aparición de una nueva generación de articulistas cuyos puentes serán Campmany y Manuel Alcántara y posteriormente Francisco Umbral quienes se inscriben en la tradición del articulismo periodístico más literario cuyo origen traza el propio César González Ruano. Otros nombres se van incorporando como Camilo José Cela, Rafael García Serrano y José Antonio Torreblanca, entre otros.

Esta generación de brillantes columnistas sufre un olvido forzado por la situación política del país. Sólo después en la transición a la democracia, estos mismos columnistas y sus herederos han llevado el asunto político a ser predominante en la natural dialéctica del periodismo de opinión.

A pesar de que en estos años estos autores evitan el tema político en sus textos y explícitamente se ocupan de asuntos de menor cuantía, ciertos rasgos de los autores de la democracia han quedado muy asentados en el periodismo de opinión tal y como hoy lo conocemos:

- Importancia de los elementos literarios y sus recursos.

- Extensión más bien breve del texto de opinión.

- Texto subjetivo ameno.

En cuanto a la transición a la democracia y las revistas de opinión, la Ley de 1966, aprobada en Cortes el 15 de marzo, de Manuel Fraga, desencadenó el proceso denominado "aperturismo" en el que, no obstante, la eliminación de la censura previa se compensaba con otros procedimientos de control.

En esos años el viejo pulso articulístico regresa a la prensa. Durante este período el diario de referencia nacional es $A B C$ aunque el periódico Pueblo disfruta de cierta implantación. Existe, por otra parte, un grupo de diarios que no sobrevive a la transición debido a su inmovilismo como es el caso de Arriba y El Alcázar. La aparición de una nueva prensa cierra el paso a publicaciones anticuadas ideológica y empresarialmente.

Se advierte en algunos periódicos la renuncia a la opinión, en favor de los géneros informativos trabajados y bien documentados. Esto ocurre en el diario Informaciones e incluso en el propio Pueblo que cuenta con una excelente escuela de periodistas jóvenes como Raúl del Pozo, Miguel Ors, Tico Medina, Jesús Hermida, José María García o Balbín.

A pesar de esta tendencia, los actores de la comunicación social no podrán dejar de implicarse en los acontecimientos de estos años. En este período, las revistas proporcionan un soporte capaz de márgenes de maniobra a los columnistas más atractivos de los que ofrece la prensa. En torno a Cuadernos para el Diálogo de tono socialista se forma una excelente nómina de periodistas, políticos y colaboradores: Jorge Esteban, Tomás de la Quadra, Amando de Miguel, Juan 
Benet, Antonio Elorza, Enrique Bustamante, Soledad Gallego, Joaquín Estefanía y Vicente Verdú. La orientación de izquierda es aún más evidente en la revista Triunfo donde se publica la columna de Manuel Vázquez Montalbán titulada "La capilla sixtina" bajo el seudónimo de Sixto Cámara. También colabora en ella Eduardo Haro Tecglen. Interviú, dirigida por Antonio Álvarez Solís, muestra desde sus inicios una decidida voluntad de opinión junto a la práctica de los grandes reportajes de investigación y de perspectiva popular.

En 1973, nace Cambio 16, fundada por Juan Tomás de Salas. En esta revista la información y la interpretación cobran protagonismo frente a la opinión. Cuenta, no obstante con columnistas importantes como Enrique Serra, Alejandro Muñoz Alonso o Luis González Seara. Buena parte de las revistas de opinión no sobreviven a la primera parte de la transición periodística a la democracia y, por tanto, otros títulos ocupan este lugar. Estas nuevas revistas van borrando poco a poco las huellas del franquismo tras la muerte del dictador.

La Constitución de 1978 incorpora en su artículo vigésimo la supresión de márgenes de acción absolutos para desplegar la libertad de expresión. Superada la transición a la democracia y con el partido socialista en el poder en los años 80 los diarios de información general sufren cierta descolocación. De este modo, periódicos como Arriba, Pueblo o la Hoja del Lunes dejan de ser publicados. Algo interesante en estos años es la importancia de la columna deportiva en los diarios de información general. Columnistas como Campmany, Alcántara o Vicente Verdú le otorgan a esta columna más relevancia que la que recibe en la prensa estrictamente deportiva.

En la segunda mitad de los setenta, aparecen los dos periódicos más significativos de la transición, El País y Diario 16. Dirigido por Juan Luis Cebrián en mayo de 1976 nace El País. Este periódico no apuesta por el columnismo, y de hecho únicamente publica "Diario de un snob" de Francisco Umbral, autor que se va a convertir, por su lenguaje original y su capacidad para renovar los cánones obsoletos del género, en el columnista más significativo de la transición democrática y en referencia para los nuevos cultivadores de este género de opinión.

El País, por tanto, se centra en el periodismo argumentativo de fondo con firmas como Julián Marías, Rafael Alberto, Fernando Chueca Goitia, Mercedes Fornica, José Luis Aranguren, Ricardo de la Cierva o Tierno Galván y a parte de estos artículos publica comentarios para completar sus informaciones con firmas como Antonio Papell o Juan Manuel Bonet.

Tampoco en su primera época Diario 16 apuesta por el columnismo. A pesar de ello cuenta con columnistas como Carmen Rico Godoy, Savater y José Luis Coll junto a un articulismo de fondo que ofrece firmas como Elías Díaz, Pedro Schwartz, Pablo Sebastián o José Luis Martínez Albertos.

Del mismo modo que en la década anterior, las revistas de los ochenta continúan llenando sus páginas de opinión. En 1985, nace Época, dirigida por 
Jaime Campmany quien imprime un cierto talante conservador a su publicación. Esta revista cuenta con la colaboración de autores como Julián Marías, Ramón Pi, Pilar Urbano, Emilio Romero, Manuel Alcántara o Ricardo de la Cierva. Tres años más tarde aparece Panorama, del grupo $\mathrm{Z}$, en la que conviven columnas de opinión (Consuelo Álvarez de Toledo, Luis Sampedro o Fernando Díaz Plaja) junto a columnas de sociedad, más próximas al revoltillo de nombres que a la opinión ( $\mathrm{M}^{\mathrm{a}}$ Eugenia Yagüe o Karmele Marchante). En 1989, nace Tribuna con talante más articulístico que columnista con firmas como Fernando Onega, Jesús Cacho, Álvaro Pombo o Andrés Aberasturi.

No es casualidad que en las revistas las firmas de prensa constituyan una referencia y un aval de ventas. Una de las consecuencias del decenio de los ochenta en la prensa española será precisamente el creciente prestigio y valoración del columnismo inspirado en el periodismo de opinión tradicional español, es decir, en el costumbrismo político sazonado en prosa satírica.

La transición al decenio último de nuestro siglo contempla la voluntad de incorporar dos títulos al sistema, El Independiente y El Sol (ambos de escasa vida) y el nacimiento de un nuevo periódico, El Mundo, que en un tiempo extraordinariamente breve, tendrá la capacidad de modificar el sistema de prensa nacional. Pedro J. Ramírez es el director del nuevo diario quien después de salir de forma conflictiva de Diario 16 arrastra con él a parte de la redacción de dicho periódico para su nuevo proyecto.

La crisis de Diario 16 provoca la salida de muchos columnistas hacia $E l$ Mundo. En la segunda mitad de la década de los noventa, la red de opinión de este diario es una de las más populares de la prensa española. En su plantilla de colaboradores cuenta con firmas como Francisco Umbral, en la última página; Antonio Burgos y Manuel Hidalgo en Cultura; Pablo Sebastián en Nacional acompañado por Pilar Urbano, Raúl Heras, Julia Navarro y Pedro Calvo Hernando; en Internacional, firma ocasionalmente Alfonso Rojo, Fernando Múgica o Xulio Ríos; en Deportes, Carlos Toro y Vicente Salaner; en Economía, Casimiro García Abadillo, y en Televisión, Carlos Boyero.

Años antes, entre 1988 y 1994 decrece de forma importante la difusión de revistas de opinión como Cambio 16, Interviú, Época, Tiempo y Tribuna a favor de un aumento en la difusión de las tres grandes cabeceras nacionales del momento: El País, $A B C$ y El Mundo. Del mismo modo que éste último, en la segunda década de los noventa, tanto El País como $A B C$ apuestan por la columna y por el prestigio de firmas que opinen en todos los ámbitos de la realidad social. Se puede afirmar que hasta ahora nunca se había escrito un periodismo de opinión en España en tal cantidad. Tras la victoria del Partido Socialista en 1982, con la que concluía la Transición democrática, se ha visto que el periodismo de opinión y sobre todo el columnismo ha conocido un auge verdaderamente extraordinario. 
La prensa en la década de los noventa se ha convertido en periodismo de discusión y debate, en definitiva, en opinión del acontecer político de los últimos años. Los periódicos actuales han devuelto la vida y la vigencia a un género periodístico, el editorial, de gran tradición como hemos podido ver en nuestro país, uno de los géneros en los que el periodismo español ha conocido sus mejores páginas.

Hoy en día el editorial es la carta de presentación y el elemento de identificación ideológica de cualquier medio de comunicación, especialmente de los escritos. Aquí radica el interés de esta investigación.

Es el género periodístico que manifiesta el punto de vista del medio sobre un determinado tema de especial relevancia para la actualidad a través de su interpretación y valoración. Es la opinión más autorizada que da el periódico sobre la interpretación de la noticia.

Según M. Lyle Spencer, el editorial es «una expresión de hechos y opiniones en un orden conciso, lógico y agradable, cuyo fin es divertir, influir en la opinión o interpretar noticias importantes de forma que se destaque su importancia para el común de los lectores» ${ }^{1}$.

El propósito del editorial es muy variado: se define un punto de vista, se crea una opinión en el público sobre un suceso determinado, se analiza y se interpreta una noticia, se relaciona un suceso concreto con otros y se establecen juicios de valor que crean actitudes en una comunidad. Forma, junto con el suelto, la nota y la glosa, lo que algunos tratadistas llaman géneros impersonales para el comentario. En palabras de Bartolomé Mostaza «la noticia da el parte diario de lo sucedido; el editorial interpreta el sentido de ese parte o previene lo que en las profundidades de la colectividad humana se está fraguando y va a estallar de un momento a otro ${ }^{2}$. Y añade el que, además de director de la Escuela Oficial de Periodismo, fuera editorialista:

«Más que una sección, por el estilo que los deportes o las críticas de teatro, cine o libros, los editoriales son una de las tres dimensiones básicas del periodismo: la dimensión de profundidad, la que, precisamente, da al periodismo autoridad y consistencia y jerarquiza en planos su contenido. Sin editoriales, el periódico quedaría reducido a mera superficie; no sería el cuerpo de la actualidad, sería su espectro.

El editorial hace trascender de su perentoria urgencia la noticia. La realidad humana -única que interesa al periodismo- habla por medio de acontecimientos, y el editorial tiene que traducir ese lenguaje de los hechos, ininteligible al no

1 RIVERS, W.: Periodismo, Prensa. Radio. Televisión. Pax. México. 1980. p. 180.

2 MOSTAZA, B.: «Editoriales», en Enciclopedia del periodismo. Barcelona- Madrid, Noguer, 1966 (cuarta edición, revisada y puesta al día) p.171. 
especializado, en términos de general comprensibilidad. Al traducir el idioma de la realidad, el editorial nos da el «significado» de lo que acaba de acontecer» ${ }^{3}$.

Al finalizar el párrafo, al hablar de «traducir el idioma de la realidad», nos remite a la idea del periodismo como interpretación de la realidad de Lorenzo Gomis:

«El editorial plantea problemas y sugiere soluciones; aprueba o reprueba lo que han hecho las autoridades o los diversos grupos o personas que influyen en la vida social y política; explica por qué es bueno o es malo lo que se ha hecho, qué consecuencias puede tener; pregunta, inquiere, urge la adopción de una solución para un problema. Recoge y expresa opiniones vigentes en el público que lo lee, y con todo ello contribuye, según hemos visto antes, a la elaboración y adopción de soluciones» ${ }^{4}$.

El editorialista se recrea en el hecho noticioso a la vez que lo destaca de los demás y apunta su trascendencia social. Desde esta perspectiva, para el profesional escribir editoriales implica:

A. Profundo conocimiento del suceso.

B. Capacidad de interpretación y análisis.

C. Expresión clara y coherente de los juicios.

Mientras que el profesor Gomis habla de un público que lee los editoriales, José Marques de Melo sostiene que, los editoriales encierran una relación de diálogo con el Estado, aunque formalmente se dirijan a la opinión pública. Él aplica esto a su país, Brasil, pero la afirmación puede ser perfectamente para toda la prensa mundial. Considera el profesor brasileño que «las instituciones periodísticas procuran decir a los dirigentes del aparato burocrático del Estado cómo les gustaría que orientasen los asuntos públicos» $»^{5}$.

La variedad temática de los editoriales es bastante amplia, aunque de manera general, según su contenido se pueden clasificar en editoriales de carácter político, económico o social. Lo cierto es que la pura selección y decisión, entre los cientos de contenidos noticiosos diarios, para establecer el orden temático de los editoriales, es una tarea complicada. Por ello, generalmente es el director del periódico quien decide el tema y señala las líneas básicas sobre las que desea se haga la reflexión.

Determinados días, sin embargo, hechos tales como un discurso del Rey, un atentado, etc., exigirán del medio la respuesta a la situación a través de un editorial. Esta es una condición de obligado cumplimiento pues aquellas publicaciones que, sistemáticamente, traten de eludir su reponsabilidad social, dedicando

3 Ibídem, p. 171.

4 GOMIS, L.: El medio media (La función política de la prensa). Barcelona, Mitre, 1988, pp. 18-19. La primera edición es de 1974.

5 MARQUeS DE MELO, J.: A opinao no jornalismo brasileiro. Petrópolis, Vozes, 1985, p. 123. 
sus editoriales a temas relativamente intrascendentes o de escasa controversia social, para así evitarse problemas con algún sector, acabarán por ser atacadas y menospreciadas por esa actitud.

Aunque cada periódico tiene su propia estructura de funcionamiento, es usual que los editorialistas presenten al director, si éste no les pide previamente algo concreto, varias posibilidades de editorializar para el número siguiente. A título meramente orientativo, indicamos los siguientes cauces para la selección temática del editorial:

1. Temas internacionales: tiene que ser de una gran importancia histórica o de directas repercusiones sobre nuestro país, para que un tema internacional merezca el preciado espacio del editorial.

2. Temas de implicación social para el lector medio: además de su índole de interés nacional, el tema escogido ha de derivar repercusiones directas y claras sobre la capa media y mayoritaria de la sociedad, a no ser que nos encontremos en una publicación definitivamente elitista.

3. Temas de conflicto, evitando el enfrentamiento: el editorial va a exigir la exposición y defensa de una opinión concreta sobre un tema de interés. En torno al mismo tiene que haber conflicto, disparidad de criterios, y nosotros hemos de optar por uno. Pero el editorial de un periódico no es una tribuna adecuada para el desencadenamiento de enfrentamientos. Éstos deben evitarse en la medida de lo posible y ser sustituidos por la imagen del diálogo y del razonamiento.

En cuanto a la influencia del editorial, ha pasado la época en la que se creía, como si fuera un dogma, que la prensa influía directamente en el público. La influencia del editorial sobre la opinión es indirecta y su alcance es, sobre todo, selectivo. Pero aunque su eco no es directo, indirectamente, el impacto puede ser poderoso. Esta forma de influencia del editorial gira en torno a su estructura redaccional.

Aunque refleje un menor índice de lectura, hay fundamentos para pensar que su eficacia relativa es más poderosa que la de cualquier otra sección del periódico si se exceptúa el comentarista de prestigio.

Por otro lado, es muy difícil ofrecer una tipología concreta y exacta de los editoriales puesto que la variedad temática de los mismos es muy amplia. Se puede decir que hay tantos editoriales como temas se pueden tratar. Luisa Santamaría ${ }^{6}$ establece tres modelos de editoriales siguiendo la tipología establecida por Martínez Albertos:

1. Editorial polémico. Su objetivo es rebatir unas posiciones contrarias y convencer por vía de la argumentación.

2. Editorial interpretativo. Suele apoyarse en el uso de razonamientos de tipo técnico. El autor somete a estudio exhaustivo los hechos y las declaraciones

6 SANTAMARIA, L.: El comentario periodístico. Los géneros persuasivos. Madrid, Paraninfo, 1990, p. 56. 
que son el tema central del editorial. Los datos técnicos y científicos son el arma más poderosa para la argumentación de estos editoriales.

3. Editorial objetivo y analítico. Se diferencia del interpretativo en que la toma de posición no es comprometida. El lenguaje es desapasionado. Apenas ofrece opinión en el texto. Este tipo de editorial no es muy frecuente en los diarios. Aparecen con motivo de cuestiones sobre las que no hay controversia o el tema es tan incipiente que carece todavía de una opinión consolidada.

Del mismo modo, Luisa Santamaría ${ }^{7}$ establece las funciones del editorial:

1. Explicar los hechos: El editorialista puede explicar a los lectores de la forma que crea más conveniente la importancia de los acontecimientos del día.

2. Ofrecer antecedentes: Además de reconocer la importancia del hecho, el editorialista debe procurar ponerlo en su contexto histórico, es decir, relacionarlo con lo que haya acontecido anteriormente.

3. Predecir el futuro: Predecir a partir del presente acontecimientos venideros a la luz de la experiencia de situaciones similares ocurridas en el pasado y siguiendo unas normas de razonamiento lógico.

4. Formular juicios. Se supone que el editorialista debe tomar partido y defender sus opiniones. La tarea del autor de editoriales es la de clarificar los hechos, analizar problemas complejos y aportar esclarecimiento sobre situaciones oscuras.

Los temas del editorial giran, preferentemente, alrededor de cuestiones políticas en el más amplio sentido de la palabra, temas de interés público general. Cada vez con más frecuencia, según Armañanzas y Díaz Noci ${ }^{8}$, aparecen temas de ámbito social y cultural. Por otra parte, no siempre los temas sobre los que versa el editorial deben ser nuevos. No obstante, el editorialista debe esforzarse por introducir matices novedosos y puntos de vista originales a temas ya conocidos.

En cuanto a su estilo, hay que tener presente que el editorial es el género de opinión que goza de menos libertad a la hora de formalizar tanto su lenguaje como su técnica y estructura. Es el género de opinión que registra mayor uniformidad y criterios fijos entre los profesionales a la hora de escribirlo. Las diferencias están en su contenido o en su orientación ideológica, no en su técnica.

En términos generales, la estructura del editorial se compone de un título que indica el tema y el cuerpo de la exposición que consta de tres partes:

$1^{a}$. Breve introducción al tema sobre el que versa el editorial.

$2^{\mathrm{a}}$. Un comentario, desarrollo del tema que constituye propiamente la opinión y aportación del periodista, porque supone la interpretación que éste hace de hechos expuestos al inicio del artículo.

7 Ibídem, p. 175.

8 ARMAÑANZAS, E; DIAZ NOCY, J. : Géneros de opinión. Periodismo y argumentación. Servicio Editorial Universidad del País Vasco, Bilbao, 1996, p. 94. 
3. La conclusión que deriva del comentario; se explican las razones por las cuales un tema se valora de una forma u otra y supone comunicar al lector un último pensamiento importante.

También hay que hacer alusión en este apartado al denominado suelto o glosa, variante menor del editorial, que goza de la misma librertad que éste, pero aparece firmado. No obstante, la firma en muchas ocasiones se trata de un pseudónimo -muchas veces claramente colectivo- o, como máximo unas iniciales ${ }^{9}$.

En opinión de Luisa Santamaría el suelto «cada día adquiere más relieve y se convierte en el fino émbolo que irrumpe en los medios para aclarar definitivamente la ideología colectiva del medio periodístico, sobre todo, en relación con algunos temas especialmente polémicos» ${ }^{10}$.

Volviendo a Bartolomé Mostaza, diremos que « el suelto es lo que un apunte, una nota marginal a este o aquel acontecimiento. Su brevedad le impide ser otra cosa que una llamada de atención para que el lector no resulte sorprendido» ${ }^{11}$.

Lo cierto es que aunque no ha sido frecuente en los estudios sobre periodismo dedicar atención al suelto o glosa su importancia es grande y de unos años a esta parte se ha notado un considerable incremento en la prensa española.

En referencia a la estructura discursiva de los Editoriales de El País tenemos que decir que la sección de «Opinión» de este diario tiene una extensión de tres páginas y es en la página que abre la sección donde se encuentran los dos o tres editoriales que cada día publica.

En general, en El País predominan los temas de ámbito nacional. Dentro de ellos, los que hablan de aspectos de la política y la economía española son los más abundantes y también los que tratan problemas de terrorismo. Además, nos encontramos con editoriales cuyo tema responde a la más estricta actualidad: ecología, iglesia, etc.

Respecto a los editoriales de ámbito internacional, en la mayoría de las ocasiones, se tratan temas económicos, políticos o sociales que tienen repercusiones directas sobre España.

En relación a su estructura, el diario El País publica diariamente dos o tres editoriales que se desarrollan en tres columnas falsas. Cuando se trata de dos editoriales, el primero suele ser un poco más extenso que el segundo. Cuando son

9 Aunque los editoriales tal como los entendemos nunca van firmados, sin embargo, podemos encontrar un comentario firmado por el director - e incluso en algunos casos por el presidente del Consejo de Administración o por el consejero delegado- que manifiesta en esos momentos el punto de vista institucional del periódico y, por tanto, tiene la consideración de editorial.

10 SANTAMARIA, L.: El comentario periodístico. Los géneros persuasivos. Ed. Paraninfo, Madrid, 1990. p 101. También en «El suelto o glosa, género editorial menor: estudio de cuatro modelos en los diarios madrileños», en Revista de Ciencias de la Información, volumen 5, Universidad Complutense de Madrid, 1988. pp. 123- 135.

11 MOSTAZA, B., Op. Cit., p. 183. 
tres los editoriales, el texto de cada uno se reparte más o menos a partes iguales y aparecen cada uno en una columna.

Por otro lado, El País se caracteriza por destacar el comienzo de los editoriales utlizando mayúsculas en las dos primeras palabras.

Si nos detenemos en el tamaño o cuerpo de la letra, éste es el mismo que el utilizado para cualquiera de las informaciones que contiene el periódico.

Si hablamos de la extensión de un editorial, ésta suele estar en función de la importancia del tema, aunque se tiende, en circunstancias normales, a un número más o menos fijo que se considera suficiente para los fines propuestos.

Esta medida varía de acuerdo con el estilo del medio. Si se compara con la extensión de los editoriales de otros diarios de difusión nacional, los de El País tienen una longitud mayor. De hecho, tienen entre 800 y 1.500 palabras (a veces más), frente a las 500 palabras de los editoriales de $A B C$ o las 400 palabras que tenían las del desaparecido Diario 16.

Hay que decir que el editorial largo y denso disminuye el interés del lector. La proporción de lectores capaces de leerlos es muy baja. El esfuerzo que requiere la lectura de editoriales más cortos es más llevadero, por lo que la proporción entre el número total de lectores y el de los que leen los editoriales resulta más equilibrada.

Los títulos utilizados por El País son frases muy cortas, normalmente de dos a cuatro palabras. Los titulares van en negrita, con un tamaño mayor que el del texto y en cursiva.

Mientras que en los titulares de las noticias se escribe lo más importante de la información, es decir, el tema, en el caso de los editoriales de El País, esta regla no se cumple. En algunas ocasiones se puede deducir el tema leyendo el titular, pero la mayoría de las veces hay que remitirse al primer párrafo del editorial.

Hay que tener en cuenta que la pequeña extensión de los títulos hace que sus responsables opten por captar y atraer la atención del lector más que por expresar claramente el tema editorial.

En cuanto a su estilo, El País se caracteriza por su estilo solemne, digno, respetuoso, correcto y sutil que va acompañado de un lenguaje preciso, claro y variado.

Aunque el vocabulario que se utiliza es accesible para todo tipo de lectores, se introducen cultismos $\mathrm{y}$, dependiendo del tema que traten los editoriales, se incluyen tecnicismos. Pueden aparecer vocablos como: catarsis, declive, desafuero, etc. En el caso de los tecnicismos nos encontramos rigor presupuestario, partidas de gasto, fondos estructurales, etc.

Sin embargo, junto a los cultismos se encuentran expresiones coloquiales y la variedad en el lenguaje se refleja tambien en la utilización de metáforas y comparaciones propias del lenguaje literario. 
El País suele hacer una mezcla de editoriales expositivos, explicativos, críticos y admonitorios porque advierten de peligros, señalan experiencias anteriores para dar ejemplo, dan argumentos, incluyen documentación y declaraciones de políticos y personas que aportan datos importantes respecto al tema tratado. Son editoriales analíticos cuyo tono es sereno, reflexivo e incluso paternal, pero sobre todo llaman al orden.

Aparte de esto, las críticas que hacen son muy repetuosas y sutiles. El País está muy lejos de ser un periódico combativo o polémico en sus editoriales. De hecho, no hace afirmaciones radicales y hace mucho uso de expresiones como: parece ser, previsiblemente, lo más razonable, deberían, parece deducirse, seguramente....

\section{Conclusiones}

- Por lo que respecta a la temática que ocupa a los editoriales de El País la política es el tema más tratado. Parece cierto cuando, en general, se dice que lo político es lo que hace que los medios sobrevivan. Concretamente es la política nacional la que más interés representa para este periódico.

- El País es el diario que hace los editoriales más extensos, entre 800 y 1.500 palabras. Aunque su extensión disminuya el interés del lector, éste puede enterarse de lo esencial del editorial leyendo el primer y último párrafo. En el primer párrafo, El País expone el tema, el problema que se va a analizar o la situación de los hechos. En el último párrafo, ofrece la conclusión o conclusiones que el periódico saca de cada tema después de haberlo analizado.

- La extensión excesiva de los editoriales de El País hace que la documentación, la aportación de datos y los argumentos y explicaciones aportadas sean muy abundantes.

- Al publicar dos o tres editoriales al día, El País tiene más posibilidades temáticas para analizar y exponer su opinión, que los periódicos que tienen un único editorial.

- La crítica de El País es bastante suave y sutil y pocas veces se expresa de forma tácita sobre algún tema.

- Los editoriales más utilizados por El País son los explicativos y los admonitorios. Con los primeros pretende asumir una posición casi pedagógica, porque intenta mostrar al lector la importancia del acontecimiento, mediante argumentos, lo más convincente posible, para que reciba una enseñanza.

- El País está lejos de ser un periódico combativo o destructivo. El tono que adopta en sus editoriales es digno y respetuoso. No suele utilizar palabras peyorativas o malsonantes.

Podemos decir que este medio mantiene una actitud más bien neutral, poco comprometida, frente a lo que editorializa. Esa neutralidad la consigue con el uso 
de un lenguaje sencillo y conciso que no ofrece muchas posibilidades para dobles sentidos o malas interpretaciones.

- Los títulos son frases muy cortas y su función principal es atraer la atención del lector. Normalmente, es preciso leer el primer párrafo del editorial para deducir su tema, ya que el título no lo aclara, es más que nada sugerente.

- Las metáforas y comparaciones propias del lenguaje literario también están presentes en los editoriales de El País. Con ello se aproximan a una estética más literaria.

- En el lenguaje combina los cultismos, las frases coloquiales y su actitud es muy comedida, es decir, tanto críticas como alabanzas son efectuadas con un tono sereno, sin emplear excesiva adjetivación.

- En definitiva, con sus editoriales El País pretende enseñar o al menos provocar la reflexión en su público más que dar soluciones a los problemas planteados. De ahí que la sutileza sea la característica principal de sus mensajes, unos mensajes que están centrados en sugerencias y reflexiones, además de la conclusión o sentencia final que suele localizarse en el último párrafo del editorial. 